\title{
Singularity of the extremal solution for supercritical biharmonic equations with power-type nonlinearity ${ }^{* \dagger}$
}

\author{
Baishun Lai ${ }^{1,2}$, Zhengxiang Yan, Yinghui Zhang \\ 1 Institute of Contemporary Mathematics, Henan University \\ Kaifeng 475004, P.R.China \\ 2 School of Mathematics and Information Science,Henan University \\ laibaishun@henu.edu.cn \\ Department of Mathematics, Hunan Institute of Science and Technology \\ Yueyang, Hunan 414006, China \\ Xinyang Vocational and Technical College, Xinyang 464000, China
}

Abstract. Let $\lambda^{*}>0$ denote the largest possible value of $\lambda$ such that

$$
\begin{cases}\Delta^{2} u=\lambda(1+u)^{p} & \text { in } \mathbb{B} \\ u=\frac{\partial u}{\partial n}=0 & \text { on } \partial \mathbb{B}\end{cases}
$$

has a solution, where $\mathbb{B}$ is the unit ball in $R^{n}$ centered at the origin, $p>\frac{n+4}{n-4}$ and $n$ is the exterior unit normal vector. We show that for $\lambda=\lambda^{*}$ this problem possesses a unique weak solution $u^{*}$, called the extremal solution. We prove that $u^{*}$ is singular when $n \geq 13$ for $p$ large enough, in which case $u^{*}(x) \leq r^{-\frac{4}{p-1}}-1$ on the unit ball and actually solve part of the open problem which [9] left.

\section{Introduction and results}

In the previous two decades, positive solutions to the second order semilinear elliptic problem

$$
\begin{cases}-\Delta u=\lambda g(u) & \text { in } \Omega, \\ u=0 & \text { on } \Omega,\end{cases}
$$

have attracted a lot of interest, see e.g. [1 5] and references therein. Here, we only mention the work by Joseph and Lundgren [2]. In their well known work, Joseph and Lundgren gave a complete characterization of all positive solutions of (1.1) in the case $g(u)=e^{u}$ or $g(u)=(1+a u)^{p}$, ap $>0, \lambda>0$ and $\Omega$ is unit ball in $R^{n}$. In particular, they found a remarkable phenomenon for $g(u)=e^{u}$ and $n>2$ : either (1.1) has at most one solution for each $\lambda$ or there is a value of $\lambda$ for which infinitely many solutions exist. In the case of a power nonlinearity the same alternative is valid if $n \geq 3$ and $p \notin\left(1, \frac{n+2}{n-2}\right]$. As a subsequent step, P.L. Lions ( 3], section 4.2 (c)) suggests to study positive solutions to systems of semilinear elliptic equations. So it is an important task to gain a deeper understanding for related higher order problems.

*Mathematics Subject Classification (2000): 35B45; 35J40

${ }^{\dagger}$ Keywords: Minimal solutions, regularity, stability, fourth order. 
In this paper we study a semilinear equation involving the bilaplacian operator and a power type nonlinearity

$$
\begin{cases}\Delta^{2} u=\lambda(1+u)^{p} & \text { in } \mathbb{B} \\ u=\frac{\partial u}{\partial n}=0 & \text { on } \partial \mathbb{B}\end{cases}
$$

where $\mathbb{B} \subset R^{n}$ is the unit ball, $\lambda>0$ is an eigenvalue parameter, $n \geq 5$ and $p \geq \frac{n+4}{n-4}$. The subcritical case $p<\frac{n+4}{n-4}$ is by now "folklore", where existence and multiplicity results are easily established by means of variational methods. For the critical case $p=\frac{n+4}{n-4}$ (under Navier boundary conditions), we refer to [6]. Recently, a lot of research on supercritical case, i.e., $p>\frac{n+4}{n-4}$, has been done and many beautiful important results have been proved. In what follows, we will summarize some of the results obtained by [7,8]. For convenience, we introduce the following notions:

Definition 1.1. We say that $u \in L^{p}(B)$ is a solution of (1.2) if $u \geq 0$ and if for all $\varphi \in C^{4}(\overline{\mathbb{B}})$ with $\varphi|\partial \mathbb{B}=| \nabla \varphi \mid \partial \mathbb{B}=0$ one has

$$
\int_{\mathbb{B}} u \Delta^{2} \varphi d x=\lambda \int_{\mathbb{B}}(1+u)^{p} \varphi d x .
$$

We call $u$ singular if $u \notin L^{\infty}(\mathbb{B})$, and regular if $u \in L^{\infty}(\mathbb{B})$.

A radial singular solution $u=u(r)$ of $(1.2)$ is called weakly singular if $\lim _{r \rightarrow 0} r^{\frac{4}{p-1}} u(r) \in$ $[0, \infty]$ exists.

Note that by standard regularity theory for the biharmonic operator, any regular solution $u$ of $(1.2)$ satisfies $u \in C^{\infty}(\overline{\mathbb{B}})$. Note also that by the positivity preserving property of $\Delta^{2}$ in the ball (see [10]) any solution of (1.2) is positive, see also [11] for a generalized statement. This property is known to fail in general domains. For this reason, we restrict ourselves to ball. Hence, the sub-and super-solution method applies as well as monotone iterative procedures.

Definition 1.2. We call a solution $u$ of (1.2) minimal if $u \leq v$ a.e. in $\mathbb{B}$ for any further solution $v$ of (1.2)

We also denote by $\lambda_{1}>0$ the first eigenvalue for the biharmonic operator with Dirichlet boundary conditions

$$
\begin{cases}\Delta^{2} \varphi_{1}=\lambda_{1} \varphi_{1} & \text { in } \mathbb{B} \\ \varphi_{1}=\frac{\partial \varphi_{1}}{\partial n}=0 & \text { on } \partial \mathbb{B} .\end{cases}
$$

It is known from the positivity preserving property and Jentzsch's (or Krein-Rutman's) theorem that $\lambda_{1}$ is isolated and simple and the corresponding eigenfunction $\varphi_{1}$ don't change sign.

Definition 1.3. We say a weak solution of (1.2) is stable (resp. semi-stable) if

$$
\mu_{1}(u)=\inf \left\{\int_{\mathbb{B}}(\Delta \varphi)^{2}-p \lambda \int_{\mathbb{B}} \varphi^{2}(1+u)^{p-1}: \phi \in H_{0}^{2}(\mathbb{B}),\|\phi\|_{L^{2}}=1\right\}
$$

is positive (resp. non-negative).

To illuminate the motivations of this paper in detail, we need the following notations which will be used throughout the paper. Set

$$
K_{0}=\frac{8(p+1)}{p-1}\left[n-\frac{2(p+1)}{p-1}\right]\left[n-\frac{4 p}{p-1}\right],
$$


and

$$
p_{c}=\frac{n+2-\sqrt{4+n^{2}-4 \sqrt{n^{2}+H_{n}}}}{n-6-\sqrt{4+n^{2}-4 \sqrt{n^{2}+H_{n}}}} \quad \text { for } n \geq 3,
$$

with $H_{n}=(n(n-4) / 4)^{2}$ and the number $p_{c}$ such that when $p=p_{c}$ then

$$
\left(\frac{4}{p-1}+4\right)\left(\frac{4}{p-1}+2\right)\left(n-2-\frac{4}{p-1}\right)\left(n-4-\frac{4}{p-1}\right)=H_{n} .
$$

Now we summarize some of the well-known results as follows:

Theorem A [7,8]. There exists $\lambda^{*} \in\left[K_{0}, \frac{\lambda_{1}}{p}\right)$ such that:

(i) For $\lambda \in\left(0, \lambda^{*}\right)$, (1.2) admits a minimal stable regular solution, denoted by $u_{\lambda}$. This solution is radially symmetric and strictly decreasing in $r=|x|$.

(ii) For $\lambda=\lambda^{*}$, (1.2) admits at least one not necessarily bounded solution, which is called extremal solution $u^{*}$

(iii) For $\lambda>\lambda^{*}$, (1.2) admits no (not even singular) solutions

Theorem B [9]. Assume that

$$
\frac{n+4}{n-4}<p<p_{c} \quad \text { if } n \geq 13, \quad \frac{n+4}{n-4}<p<\infty \text { if } 5 \leq n \leq 12
$$

Then, $u^{*}$ is regular.

From Theorem B, we know that the extremal solution of (1.2) is regular for a certain range of $p$ and $n$. At the same time, they left a open problem: if

$$
n \geq 13 \text { and } p \geq p_{c}
$$

is $u^{*}$ singular?

In this paper, by constructing a semi-stable singular $H_{0}^{2}(\mathbb{B})$ - weak sub-solution of (1.2), we prove that, if $p$ is large enough, the extremal solution is singular for dimensions $n \geq 13$ and complete part of the above open problem. Our result is stated as follows:

Theorem 1.1. There exists $p_{0}>1$ large enough such that for $p \geq p_{0}$, the unique extremal solution of (1.2) is singular for dimensions $n \geq 13$, in which case $u^{*} \leq|x|^{-\frac{4}{p-1}}-1$ on the unit ball.

From the technical point of view, one of the obstacle is the well-known difficulty of extracting energy estimates for solutions of fourth order problems from their stability properties. Besides, for the corresponding second order problem (1.1), the starting point was an explicit singular solution for a suitable eigenvalue parameter $\lambda$ which turned out to play a fundamental role for the shape of the corresponding bifurcation diagram, see [12]. When turning to the biharmonic problem (1.2) the second boundary condition $\frac{\partial u}{\partial n}=0$ prevents to find an explicit singular solution. This means that the method used to analyze the regularity of the extremal solution for second order problem could not carry to the corresponding problem for (1.2). In this paper, we, in order to overcome the second obstacle, use improved and non standard Hardy-Rellich inequalities recently established by Ghoussoub-Moradifam in [13] to construct a semi-stable singular $H_{0}^{2}(\mathbb{B})$ - weak subsolution of (1.2). 
This paper is organized as follows. In the next section, some preliminaries are reviewed. In Section 3, we will show that the extremal solution $u^{*}$ in dimensions $n \geq 13$ is singular by constructing a semi-stable singular $H_{0}^{2}(\mathbb{B})$ - weak sub-solution of (1.2).

\section{Preliminaries}

First we give some comparison principles which will be used throughout the paper.

Lemma 2.1. (Boggio's principle, [10]) If $u \in C^{4}\left(\overline{\mathbb{B}}_{R}\right)$ satisfies

$$
\begin{cases}\Delta^{2} u \geq 0 & \text { in } \mathbb{B}_{R} \\ u=\frac{\partial u}{\partial n}=0 & \text { on } \quad \partial \mathbb{B}_{R}\end{cases}
$$

then $u \geq 0$ in $\mathbb{B}_{R}$.

Lemma 2.2. Let $u \in L^{1}\left(\mathbb{B}_{R}\right)$ and suppose that

$$
\int_{\mathbb{B}_{R}} u \Delta^{2} \varphi \geq 0
$$

for all $\varphi \in C^{4}\left(\overline{\mathbb{B}}_{R}\right)$ such that $\varphi \geq 0$ in $\mathbb{B}_{R},\left.\varphi\right|_{\partial \mathbb{B}_{R}}=\left.\frac{\partial \varphi}{\partial n}\right|_{\partial \mathbb{B}_{R}}=0$. Then $u \geq 0$ in $\mathbb{B}_{R}$. Moreover $u \equiv 0$ or $u>0$ a.e., in $\mathbb{B}_{R}$.

For a proof see Lemma 17 in [11].

Lemma 2.3. If $u \in H^{2}\left(\mathbb{B}_{R}\right)$ is radial, $\Delta^{2} u \geq 0$ in $\mathbb{B}_{R}$ in the weak sense, that is

$$
\int_{\mathbb{B}_{R}} \Delta u \Delta \varphi \geq 0 \quad \forall \varphi \in C_{0}^{\infty}\left(\mathbb{B}_{R}\right), \varphi \geq 0
$$

and $\left.u\right|_{\partial \mathbb{B}_{R}} \geq 0,\left.\frac{\partial u}{\partial n}\right|_{\partial \mathbb{B}_{R}} \leq 0$, then $u \geq 0$ in $\mathbb{B}_{R}$.

Proof. For the sake of completeness, we include a brief proof here. We only deal with the case $R=1$ for simplicity. Solve

$$
\begin{cases}\Delta^{2} u_{1}=\Delta^{2} u & \text { in } \mathbb{B} \\ u_{1}=\frac{\partial u_{1}}{\partial n}=0 & \text { on } \quad \partial \mathbb{B}\end{cases}
$$

in the sense $u_{1} \in H_{0}^{2}(\mathbb{B})$ and $\int_{\mathbb{B}} \Delta u_{1} \Delta \varphi=\int_{\mathbb{B}} \Delta u \Delta \varphi$ for all $\varphi \in C_{0}^{\infty}(\mathbb{B})$. Then $u_{1} \geq 0$ in $\mathbb{B}$ by lemma 2.2 .

Let $u_{2}=u-u_{1}$ so that $\Delta^{2} u_{2}=0$ in $\mathbb{B}$. Define $f=\Delta u_{2}$. Then $\Delta f=0$ in $\mathbb{B}$ and since $f$ is radial we find that $f$ is a constant. It follows that $u_{2}=a r^{2}+b$. Using the boundary conditions we deduce $a+b \geq 0$ and $a \leq 0$, which imply $u_{2} \geq 0$.

Now we give a notion of $H_{0}^{2}(\mathbb{B})$ - weak solutions, which is an intermediate class between classical and weak solutions.

Definition 2.1. We say that $u$ is a $H_{0}^{2}(\mathbb{B})$ - weak solution of $(1.2)$ if $(1+u)^{p} \in L^{1}(\mathbb{B})$ and if

$$
\int_{\mathbb{B}} \Delta u \Delta \phi=\lambda \int_{\mathbb{B}} \phi(1+u)^{p}, \quad \forall \phi \in C^{4}(\overline{\mathbb{B}}) \cap H_{0}^{2}(\mathbb{B}) .
$$

We say that $u$ is a $H_{0}^{2}(\mathbb{B})$ - weak super-solution (resp. $H_{0}^{2}(\mathbb{B})$ - weak sub-solution) of (1.2) if for $\phi \geq 0$ the equality is replaced with $\geq$ (resp. $\leq$ ) and $u \geq 0$ (resp. $\leq$ ), $\frac{\partial u}{\partial n} \leq 0$ (resp. $\geq$ ) on $\partial \mathbb{B}$.

We also need the following comparison principle. 
Lemma 2.4. Let $u_{1}, u_{2} \in H^{2}\left(\mathbb{B}_{R}\right)$ with $\left(1+u_{1}\right)^{p},\left(1+u_{2}\right)^{p} \in L^{1}\left(\mathbb{B}_{R}\right)$. Assume that $u_{1}$ is stable and

$$
\Delta^{2} u_{1} \leq \lambda\left(1+u_{1}\right)^{p} \text { in } \mathbb{B}_{R}
$$

in the $H^{2}\left(\mathbb{B}_{R}\right)$ - weak sense, i.e.,

$$
\int_{\mathbb{B}_{R}} \Delta u_{1} \Delta \phi \leq \lambda \int_{\mathbb{B}_{R}}\left(1+u_{1}\right)^{p} \phi, \quad \forall \phi \in C_{0}^{\infty}\left(\mathbb{B}_{R}\right), \phi \geq 0
$$

and $\Delta^{2} u_{2} \geq \lambda\left(1+u_{2}\right)^{p} \quad$ in $\mathbb{B}_{R}$ in the similar weak sense. Suppose also

$$
\left.u_{1}\right|_{\partial \mathbb{B}_{R}}=\left.u_{2}\right|_{\partial \mathbb{B}_{R}} \text { and }\left.\frac{\partial u_{1}}{\partial n}\right|_{\partial \mathbb{B}_{R}}=\left.\frac{\partial u_{2}}{\partial n}\right|_{\partial \mathbb{B}_{R}} .
$$

Then

$$
u_{1} \leq u_{2} \quad \text { in } \mathbb{B}_{R}
$$

Proof. Define $\omega:=u_{1}-u_{2}$. Then by the Moreau decomposition 14 for the biharmonic operator, there exist $\omega_{1}, \omega_{2} \in H_{0}^{2}\left(\mathbb{B}_{R}\right)$, with $\omega=\omega_{1}+\omega_{2}, \omega_{1} \geq 0$ a.e., $\Delta^{2} \omega_{2} \leq 0$ in the $H_{0}^{2}\left(\mathbb{B}_{R}\right)$ - weak sense and

$$
\int_{\mathbb{B}_{R}} \Delta \omega_{1} \Delta \omega_{2}=0
$$

By Lemma 1.1, we have that $\omega_{2} \leq 0$ a.e. in $\mathbb{B}_{R}$.

Given now $0 \leq \varphi \in C_{0}^{\infty}\left(\mathbb{B}_{R}\right)$, we have that

$$
\int_{\mathbb{B}_{R}} \Delta \omega \Delta \varphi \leq \lambda \int_{\mathbb{B}_{R}}\left(f\left(u_{1}\right)-f\left(u_{2}\right)\right) \varphi
$$

where $f(u)=(1+u)^{p}$. Since $u$ is semi-stable and by density one has

$$
\lambda \int_{\mathbb{B}_{R}} f^{\prime}(u) \omega_{1}^{2} \leq \lambda \int_{\mathbb{B}_{R}}\left(\Delta \omega_{1}\right)^{2}=\lambda \int_{\mathbb{B}_{R}} \Delta \omega \Delta \omega_{1} \leq \lambda \int_{\mathbb{B}_{R}}\left(f\left(u_{1}\right)-f\left(u_{2}\right)\right) \omega_{1} .
$$

Since $\omega_{1} \geq \omega$, one also has

$$
\int_{\mathbb{B}_{R}} f^{\prime}(u) \omega \omega_{1} \leq \int_{\mathbb{B}_{R}}\left(f\left(u_{1}\right)-f\left(u_{2}\right)\right) \omega_{1}
$$

which once re-arrange gives

$$
\int_{\mathbb{B}_{R}} \tilde{f} \omega_{1} \geq 0
$$

where $\tilde{f}\left(u_{1}\right)=f\left(u_{1}\right)-f\left(u_{2}\right)-f^{\prime}\left(u_{1}\right)\left(u_{1}-u_{2}\right)$. The strict convexity of $f$ gives $\tilde{f} \leq 0$ and $\tilde{f}<0$ whenever $u \neq U$. Since $\omega_{1} \geq 0$ a.e. in $\mathbb{B}_{R}$, one sees that $\omega \leq 0$ a.e. in $\mathbb{B}_{R}$. The inequality $u_{1} \leq u_{2}$ a.e. in $\mathbb{B}_{R}$ is then established.

The following variant of lemma 2.4 also holds:

Lemma 2.5. Let $u_{1}, u_{2} \in H^{2}\left(\mathbb{B}_{R}\right)$ be radial with $\left(1+u_{1}\right)^{p},\left(1+u_{2}\right)^{p} \in L^{1}\left(\mathbb{B}_{R}\right)$. Assume $\Delta^{2} u_{1} \leq \lambda\left(1+u_{1}\right)^{p} \quad$ in $\mathbb{B}_{R}$ in the sense of (2.1) and and $\Delta^{2} u_{2} \geq \lambda\left(1+u_{2}\right)^{p}$ in $\mathbb{B}_{R}$. Suppose $\left.u_{1}\right|_{\partial \mathbb{B}_{R}} \leq\left. u_{2}\right|_{\partial \mathbb{B}_{R}}$ and $\left.\frac{\partial u_{1}}{\partial n}\right|_{\partial \mathbb{B}_{R}} \geq\left.\frac{\partial u_{2}}{\partial n}\right|_{\partial \mathbb{B}_{R}}$ and suppose also that $u_{1}$ is semi-stable. Then $u_{1} \leq u_{2}$ in $\mathbb{B}_{R}$. 
Proof. We solve for $\hat{u} \in H_{0}^{2}(\mathbb{B})$ such that

$$
\int_{\mathbb{B}_{R}} \Delta \hat{u} \Delta \phi=\int_{\mathbb{B}_{R}} \Delta\left(u_{1}-u_{2}\right) \Delta \phi \quad \forall \phi \in C_{0}^{\infty}\left(\mathbb{B}_{R}\right) .
$$

By Lemma 2.3 it follows that $\hat{u} \geq u_{1}-u_{2}$. Next we apply the Moreau decomposition to $\hat{u}$, that is $\hat{u}=w+v$ with $w, v \in H_{0}^{2}\left(\mathbb{B}_{R}\right), w \geq 0, \Delta^{2} v \leq 0$ in $\mathbb{B}_{R}$ and $\int_{\mathbb{B}_{R}} \Delta w \Delta v=0$. Then the argument follows that of Lemma 2.4.

Lemma 2.6. Let $u$ be a semi-stable $H_{0}^{2}(\mathbb{B})$ - weak solution of (1.2). Assume $U$ is a $H_{0}^{2}(\mathbb{B})$ - super-solution of (1.2). Then if $u$ is a classical solution and $\mu_{1}(u)=0$, we have $u=U$.

Proof. Since $u$ is a classical solution, it is easy to see that the infimum in $\mu_{1}(u)$ is attained at some $\varphi$. The function $\varphi$ is then the first eigenfunction of $\Delta^{2}-\lambda f^{\prime}(u)$ in $H_{0}^{2}(\mathbb{B})$, where $f(u)=(1+u)^{p}$. Now we show that $\phi$ is of fixed sign. Using the Moreau decomposition, one has $\phi=\phi_{1}+\phi_{2}$ where $\phi_{i} \in H_{0}^{2}(\mathbb{B})$ for $i=1,2, \phi_{1} \geq 0, \int_{\mathbb{B}} \Delta \phi_{1} \Delta \phi_{2}=0$ and $\Delta^{2} \phi_{2} \leq 0$ in the $H_{0}^{2}(\mathbb{B})$ - weak sense. If $\phi$ changes sign, then $\phi_{1} \not \equiv 0$ and $\phi_{2}<0$ in $\mathbb{B}$. We can write now:

$$
0=\mu_{1}(u) \leq \frac{\int_{\mathbb{B}}\left(\Delta\left(\phi_{1}-\phi_{2}\right)\right)^{2}-\lambda f^{\prime}(u)\left(\phi_{1}-\phi_{2}\right)^{2}}{\int_{\mathbb{B}}\left(\phi_{1}-\phi_{2}\right)^{2}}<\frac{\int_{\mathbb{B}}(\Delta \phi)^{2}-\lambda f^{\prime}(u) \phi^{2}}{\int_{\mathbb{B}} \phi^{2}}=\mu_{1}(u)
$$

in view of $\phi_{1} \phi_{2}<-\phi_{1} \phi_{2}$ in a set of positive measure, leading to a contradiction.

So we can assume $\phi \geq 0$, and by the Boggio's principle we have $\phi>0$ in $\mathbb{B}$. For $0 \leq t \leq 1$ define

$$
g(t)=\int_{\mathbb{B}} \Delta(t U+(1-t) u) \Delta \phi-\lambda \int_{\mathbb{B}} f(t U+(1-t) u) \phi,
$$

where $\phi$ is the above first eigenfunction. Since $f$ is convex one sees that

$$
g(t) \geq \lambda \int_{\mathbb{B}}[t f(U)+(1-t) f(u)-f(t U+(1-t) u)] \phi \geq 0
$$

for every $t \geq 0$. Since $g(0)=0$ and

$$
g^{\prime}(0)=\int_{\mathbb{B}} \Delta(U-u) \Delta \phi-\lambda f^{\prime}(u)(U-u) \phi=0
$$

we get that

$$
g^{\prime \prime}(0)=-\lambda \int_{\mathbb{B}} f^{\prime \prime}(u)(U-u)^{2} \phi \geq 0 .
$$

Since $f^{\prime \prime}(u) \phi>0$ in $\mathbb{B}$, we finally get that $U=u$ a.e. in $\mathbb{B}$.

From this lemma, we immediately obtain:

Corollary 2.1 (i) When $u^{*}$ is a classical solution, then $\mu_{1}\left(u^{*}\right)=0$ and $u^{*}$ is the unique $H_{0}^{2}(\mathbb{B})$ - weak solution of (1.2);

(ii) If $v$ is a singular semi-stable $H_{0}^{2}(\mathbb{B})$ - weak solution of (1.2), then $v=u^{*}$ and $\lambda=\lambda^{*}$.

Proof. (i) Since the function $u^{*}$ is a classical solution, and by the Implicit Function Theorem we have that $\mu_{1}\left(u^{*}\right)=0$ to prevent the continuation of the minimal branch beyond $\lambda^{*}$. By Lemma 2.4, $u^{*}$ is then the unique $H_{0}^{2}(\mathbb{B})$ - weak solution of (1.2). 
(ii) Assume now that $v$ is a singular semi-stable $H_{0}^{2}(\mathbb{B})-$ weak solution of (1.2). If $\lambda<\lambda^{*}$, then by the uniqueness of the semi-stable solution, we have $v=u_{\lambda}$. So $v$ is not singular and a contradiction arises. By Theorem A (iii) we have that $\lambda=\lambda^{*}$. Since $v$ is a semi-stable $H_{0}^{2}(\mathbb{B})$ - weak solution of $(1.2)$ and $u^{*}$ is a $H_{0}^{2}(\mathbb{B})$ - weak super-solution of (1.2), we can apply Lemma 2.4 to get $v \leq u^{*}$ a.e. in $\mathbb{B}$. Since $u^{*}$ is a semi-stable solution too, we can reverse the roles of $v$ and $u^{*}$ in Lemma 2.5 to see that $v \geq u^{*}$ a.e. in $\mathbb{B}$. So equality $v=u^{*}$ holds and the proof is complete.

\section{Proof of Theorem 1.1}

Inspired by the work of [16], we will first show the following upper bound on $u^{*}$

Lemma 3.1. If $n \geq 13$ and $p>p_{c}$, then $u^{*} \leq|x|^{-\frac{4}{p-1}}-1$.

Proof. Recall from Theorem A that $K_{0}<\lambda^{*}$. We now claim that $u_{\lambda} \leq \tilde{u}:=|x|^{-\frac{4}{p-1}}-1$ for all $\lambda \in\left(K_{0}, \lambda^{*}\right)$. Indeed, fix such a $\lambda$ and assume by contradiction that

$$
R_{1}:=\inf \left\{0 \leq R \leq 1: u_{\lambda}<\bar{u} \quad \text { in the interval }(R, 1)\right\}>0 .
$$

From the boundary conditions, one has that

$$
u_{\lambda}(r)<\tilde{u}(r) \text { as } r \rightarrow 1^{-} .
$$

Hence,

$$
0<R_{1}<1, u_{\lambda}\left(R_{1}\right)=\tilde{u}\left(R_{1}\right) \text { and } u_{\lambda}^{\prime}\left(R_{1}\right) \leq \tilde{u}^{\prime}\left(R_{1}\right) .
$$

Now consider the following problem

$$
\begin{cases}\Delta^{2} u=K_{0}(1+u)^{p} & \text { in } \mathbb{B}_{R_{1}} \\ u=u_{\lambda}\left(R_{1}\right) & \text { on } \partial \mathbb{B}_{R_{1}} \\ \frac{\partial u}{\partial n}=u_{\lambda}^{\prime}\left(R_{1}\right) & \text { on } \partial \mathbb{B}_{R_{1}}\end{cases}
$$

Then $u_{\lambda}$ is a super-solution to above problem while $\tilde{u}$ is a sub-solution to the same problem. Moreover for $n \geq 13$, we have

$$
p K_{0} \leq H_{n}:=\frac{n^{2}(n-4)^{2}}{16}
$$

and

$$
\int_{\mathbb{B}_{R_{1}}}(\Delta \phi)^{2} \geq H_{n} \int_{\mathbb{B}_{R_{1}}} \frac{\phi^{2}}{|x|^{4}} d x \geq p K_{0} \int_{\mathbb{B}_{R_{1}}}(1+\tilde{u})^{p-1} .
$$

So $\tilde{u}$ is semi-stable and we deduce that $u_{\lambda}>\tilde{u}$ by the Lemma 2.4, and a contradiction arises in view of the fact

$$
\left|u_{\lambda}\right|_{L^{\infty}\left(\mathbb{B}_{R_{1}}\right)}<\infty \text {, and }|\tilde{u}|_{L^{\infty}\left(\mathbb{B}_{R_{1}}\right)}=\infty
$$

The proof is done.

In order to prove Theorem 1.1, we will need a suitable Hardy-Rellich type inequality which was established by Ghoussoub-Moradifam in [13]. It is stated as follows:

Lemma 3.2. Let $n \geq 5$ and $\mathbb{B}$ be the unit ball in $\mathbb{R}^{n}$. Then there exists $C>0$, such that the following improved Hardy-Rellich inequality holds for all $\varphi \in H_{0}^{2}(\mathbb{B})$ :

$$
\int_{\mathbb{B}}(\Delta \varphi)^{2} d x \geq \frac{n^{2}(n-4)^{2}}{16} \int_{\mathbb{B}} \frac{\varphi^{2}}{|x|^{4}} d x+C \int_{\mathbb{B}} \varphi^{2} d x
$$


Lemma 3.3. Let $n \geq 5$ and $\mathbb{B}$ be the unit ball in $\mathbb{R}^{n}$. Then the following improved Hardy-Rellich inequality holds for all $\varphi \in H_{0}^{2}(\mathbb{B})$ :

$$
\begin{aligned}
\int_{\mathbb{B}}(\Delta \varphi)^{2} d x & \geq \frac{(n-2)^{2}(n-4)^{2}}{16} \int_{\mathbb{B}} \frac{\varphi^{2} d x}{\left(|x|^{2}-0.9|x|^{\frac{n}{2}+1}\right)\left(|x|^{2}-|x|^{\frac{n}{2}}\right)} \\
& +\frac{(n-1)(n-4)^{2}}{4} \int_{\mathbb{B}} \frac{\varphi^{2} d x}{|x|^{2}\left(|x|^{2}-|x|^{\frac{n}{2}}\right)} .
\end{aligned}
$$

As a consequence, the following improvement of the classical Hardy-Rellich inequality holds:

$$
\int_{\mathbb{B}}(\Delta \varphi)^{2} d x \geq \frac{n^{2}(n-4)^{2}}{16} \int_{\mathbb{B}} \frac{\varphi^{2}}{|x|^{2}\left(|x|^{2}-|x|^{\left.\frac{n}{2}\right)}\right.} .
$$

We now give the following lemma which is crucial for the proof of the Theorem 1.1.

Lemma 3.4. Suppose there exist $\lambda^{\prime}>0$ and a radial function $u \in H^{2}(\mathbb{B}) \cap W_{\text {loc }}^{4, \infty}(\mathbb{B} \backslash\{0\})$ such that $u \notin L^{\infty}(\mathbb{B})$ and

$$
\Delta^{2} u \leq \lambda^{\prime}(1+u)^{p} \text { for } 0<r<1 ; \quad u(1)=u^{\prime}(1)=0
$$

and

$$
p \beta \int_{\mathbb{B}} \varphi^{2}(1+u)^{p+1} \leq \int_{\mathbb{B}}(\Delta \varphi)^{2} \quad \text { for all } \varphi \in H_{0}^{2}(\mathbb{B})
$$

for either $\beta>\lambda^{\prime}$ or $\beta=\lambda^{\prime}=\frac{H_{n}}{p}$. Then $u^{*}$ is singular and

$$
\lambda^{*} \leq \lambda^{\prime}
$$

Proof. First, we prove $\lambda^{*} \leq \lambda^{\prime}$. Note that the stability and $u \in L_{\text {loc }}^{\infty}(\mathbb{B} \backslash\{0\})$ yield to $(1+u)^{p} \in L^{1}(\mathbb{B})$, we easily see that $u$ is a weak sub-solution of (1.2). If now $\lambda^{\prime}<\lambda^{*}$, by Lemma $2.5, u$ would necessarily be below the minimal solution $u_{\lambda^{\prime}}$, which is a contraction since $u$ is singular while $u_{\lambda^{\prime}}$ is regular.

Suppose first that $\beta=\lambda^{\prime}=\frac{H_{n}}{p}$ and that $n \geq 13$. From the above we have $\lambda^{*} \leq \frac{H_{n}}{p}$, we get from Lemma 3.1 and the improved Hardy-Rellich inequality that there exists $C>0$ so that for all $\phi \in H_{0}^{2}(\mathbb{B})$

$$
\int_{\mathbb{B}}(\Delta \phi)^{2}-p \lambda^{*} \int_{\mathbb{B}} \phi^{2}\left(1+u^{*}\right)^{p+1} \geq \int_{\mathbb{B}}(\Delta \phi)^{2}-H_{n} \int_{\mathbb{B}} \frac{\phi^{2}}{|x|^{4}} \geq C \int_{\mathbb{B}} \phi^{2}
$$

It follows that $\mu_{1}\left(u^{*}\right)>0$ and $u^{*}$ must therefore be singular since otherwise, one could use the Implicit Function Theorem to continue the minimal branch beyond $\lambda^{*}$

Suppose now that $\beta>\lambda^{\prime}$ and let $\frac{\lambda^{\prime}}{\beta_{1}}<\gamma<1$ and $\alpha:=\left(\frac{\gamma \lambda^{*}}{\lambda^{\prime}}\right)^{\frac{1}{p+1}}$ and define $\bar{u}:=$ $\alpha^{-1}(1+u)-1$. We claim that

$$
u^{*} \leq \bar{u} \quad \text { in } \mathbb{B} .
$$

To prove this, we shall show that for every $\lambda<\lambda^{*}$

$$
u_{\lambda} \leq \bar{u} \quad \text { in } \mathbb{B}
$$


Indeed, we have

$$
\Delta^{2} \bar{u}=\alpha \Delta^{2} u \leq \alpha \lambda^{\prime}(1+u)^{p}=\alpha^{p+1} \lambda^{\prime}(1+\bar{u})^{p} .
$$

Now by the choice of $\alpha$, we have $\alpha^{p+1} \lambda^{\prime}<\lambda^{*}$. To prove (3.3), it suffices to prove it for $\alpha^{p+1} \lambda^{\prime}<\lambda<\lambda^{*}$. Fix such $\lambda$ and assume that (3.3) is not true. Then

$$
\Lambda=\left\{0 \leq R \leq 1 \mid u_{\lambda}(R)>\bar{u}(R)\right\}
$$

is non-empty. Since $\bar{u}(1)=\alpha^{-1}-1>0=u_{\lambda}(1)$, we have $0<R_{1}<1, u_{\lambda}\left(R_{1}\right)=\bar{u}\left(R_{1}\right)$, and $u_{\lambda}^{\prime}\left(R_{1}\right) \leq \bar{u}^{\prime}\left(R_{1}\right)$. Now consider the following problem

$$
\begin{cases}\Delta^{2} u=\lambda(1+u)^{p} & \text { in } \mathbb{B}_{R_{1}} \\ u=u_{\lambda}\left(R_{1}\right) & \text { on } \partial \mathbb{B}_{R_{1}} \\ \frac{\partial u}{\partial n}=u_{\lambda}^{\prime}\left(R_{1}\right) & \text { on } \partial \mathbb{B}_{R_{1}}\end{cases}
$$

Then $u_{\lambda}$ is a solution to above problem while $\bar{u}$ is a sub-solution to the same problem. Moreover $\bar{u}$ is stable since $\lambda<\lambda^{*}$ and

$$
p \lambda(1+\bar{u})^{p+1} \leq p \lambda^{*} \alpha^{-(p+1)}(1+u)^{p+1}=p \lambda^{\prime} \gamma^{-1}(1+u)^{p+1}<p \beta_{1}(1+u)^{p+1},
$$

we deduce $\bar{u} \leq u_{\lambda}$ in $\mathbb{B}_{R_{1}}$, which is impossible, since $\bar{u}$ is singular while $u_{\lambda}$ is smooth. This establishes (3.2). From (3.2) and the above inequalities, we have

$$
p \lambda^{*}\left(1+u^{*}\right)^{p+1} \leq p \lambda^{\prime} \gamma^{-1}(1+u)^{p+1}<p \beta_{1}(1+u)^{p+1} .
$$

Thus

$$
\inf _{\varphi \in C_{0}^{\infty}(\mathbb{B})} \frac{\int_{\mathbb{B}}(\Delta \varphi)^{2}-p \lambda^{*} \varphi^{2}\left(1+u^{*}\right)^{p+1}}{\int_{\mathbb{B}} \varphi^{2}}>0 .
$$

This is not possible if $u^{*}$ is a smooth function by the Implicit Theorem.

Proof Theorem 1.1 Uniqueness and the upper bound estimate of the extremal solution $u^{*}$ have been proven by Corollary 3.1 and Lemma 3.1. Now we only prove that $u^{*}$ is a singular solution of (1.1) for $n \geq 13$, in order to achieve this, we shall find a singular $H$-weak sub-solution of $(1.1)$, denote by $\omega_{m}(r)$, which is stable, according to the Lemma 3.4 .

Choosing

$$
\omega_{m}=a_{1} r^{-\frac{4}{p-1}}+a_{2} r^{m}-1, \quad K_{0}=\frac{8(p+1)}{p-1}\left[n-\frac{2(p+1)}{p-1}\right]\left[n-\frac{4 p}{p-1}\right]
$$

since $\omega(1)=\omega^{\prime}(1)=0$, we have

$$
a_{1}=\frac{m}{m+\frac{4}{p-1}} \quad a_{2}=\frac{\frac{4}{p-1}}{m+\frac{4}{p-1}}
$$

For any $m$ fixed, when $p \rightarrow+\infty$, we have

$$
a_{1}=1-\frac{4}{(p-1) m}+o\left(p^{-1}\right), \quad a_{2}=1-a_{1}=\frac{4}{(p-1) m}+o\left(p^{-1}\right)
$$

and

$$
K_{0}=\frac{8(n-2)(n-4)}{p}+o\left(p^{-1}\right)
$$


Note that

$$
\begin{aligned}
& \lambda^{\prime} K_{0}\left(1+\omega_{m}(r)\right)^{p}-\Delta^{2} \omega_{m}(r)=\lambda^{\prime} K_{0}\left(1+\omega_{m}(r)\right)^{p}-a_{1} K_{0} r^{-\frac{4 p}{p-1}}-a_{2} K_{1} r^{m-4} \\
= & \lambda^{\prime} K_{0}\left(a_{1} r^{-\frac{4}{p-1}}+a_{2} r^{m}\right)^{p}-a_{1} K_{0} r^{-\frac{4 p}{p+1}}-a_{2} K_{1} r^{m-4} \\
= & K_{0} r^{-\frac{4 p}{p-1}}\left[\lambda^{\prime}\left(a_{1}+a_{2} r^{m+\frac{4}{p-1}}\right)^{p}-a_{1}-a_{2} K_{1} K_{0}^{-1} r^{\frac{4 p}{p-1}+m-4}\right] \\
= & K_{0} r^{-\frac{4 p}{p-1}}\left[\lambda^{\prime}\left(a_{1}+a_{2} r^{m+\frac{4}{p-1}}\right)^{p}-a_{1}-a_{2} K_{1} K_{0}^{-1} r^{m-\frac{4}{p-1}}\right] \\
= & K_{0} r^{-\frac{4 p}{p-1}}\left(a_{1}+a_{2} r^{m+\frac{4}{p-1}}\right)^{p}\left[\lambda^{\prime}-H\left(r^{m+\frac{4}{p-1}}\right)\right]
\end{aligned}
$$

with

$$
H(x)=\left(a_{1}+a_{2} x\right)^{p}\left[a_{1}+a_{2} K_{1} K_{0}^{-1} x\right], K_{1}=m(m-2)(m+n-2)(m+n-4)
$$

(1) Let $m=2$ and $n \geq 32$, then we can prove that

$$
\sup _{[0,1]} H(x)=H(0)=a_{1}^{1-p} \longrightarrow e^{2} \text { as } p \longrightarrow+\infty \text {. }
$$

So $(3.4) \geq 0$ is valid as long as

$$
\lambda^{\prime}=e^{2}
$$

At the same time, we have ( since $a_{1}+a_{2} r^{m+\frac{4}{p-1}} \leq a_{1}+a_{2} \leq 1$ in $[0,1]$ )

$$
\frac{n^{2}(n-4)^{2}}{16} \frac{1}{r^{4}}-p \beta_{n} r^{-4}\left(a_{1}+a_{2} r^{2+\frac{4}{p-1}}\right)^{p-1} \geq r^{-4}\left[\frac{n^{2}(n-4)^{2}}{16}-p \beta\right] \text {. }
$$

Let $\beta=\left(\lambda^{\prime}+\varepsilon\right) K_{0}$, where $\varepsilon$ is arbitrary sufficient small, we need finally here

$$
\frac{n^{2}(n-4)^{2}}{16}-p \beta=\frac{n^{2}(n-4)^{2}}{16}-p\left(\lambda^{\prime}+\varepsilon\right) K_{0}>0 .
$$

For that, it is sufficient to have for $p \longrightarrow+\infty$

$$
\frac{n^{2}(n-4)^{2}}{16}-8\left(e^{2}+\varepsilon\right)(n-2)(n-4)+o\left(\frac{1}{p}\right)>0 .
$$

So (3.6) $\geq 0$ holds only for $n \geq 32$ when $p \longrightarrow+\infty$. Moreover, for $p$ large enough

$$
8 e^{2}(n-2)(n-4) \int_{\mathbb{B}} \varphi^{2}\left(1+\omega_{2}\right)^{p+1} \leq H_{n} \int_{\mathbb{B}} \frac{\varphi^{2}}{|x|^{4}} \leq \int_{\mathbb{B}}|\Delta \varphi|^{2}
$$

Thus it follows from Lemma 3.4 that $u^{*}$ is singular with $\lambda^{\prime}=e^{2} K_{0}, \beta=\left(e^{2} K_{0}+\varepsilon(n, p)\right)$ and $\lambda^{*} \leq e^{2} K_{0}$.

(2) Assume $13 \leq n \leq 31$. We shall show that $u=\omega_{3.5}$ satisfies the assumptions of Lemma 5.4 for each dimension $13 \leq n \leq 31$. Using Maple, for each dimension $13 \leq n \leq 31$ one can verify that inequality (3.4) $\geq 0$ holds for the $\lambda^{\prime}$ given by Table 1 . Then, by using Maple again, we show that there exists $\beta>\lambda^{\prime}$ such that

$$
\begin{aligned}
\frac{(n-2)^{2}(n-4)^{2}}{16} & \frac{1}{\left(|x|^{2}-0.9|x|^{\frac{n}{2}+1}\right)\left(|x|^{2}-|x|^{\frac{n}{2}}\right)} \\
+ & \frac{(n-1)(n-4)^{2}}{4} \frac{1}{|x|^{2}\left(|x|^{2}-|x|^{\frac{n}{2}}\right)} \geq p \beta\left(1+w_{3.5}\right)^{p+1} .
\end{aligned}
$$

The above inequality and and improved Hardy-Rellich inequality (5.0) guarantee that the stability condition (5.2) holds for $\beta>\lambda^{\prime}$. Hence by Lemma 3.4 the extremal solution is singular for $13 \leq n \leq 31$ the value of $\lambda^{\prime}$ and $\beta$ are shown in Table 1 . 
Remark 1 The values of $\lambda^{\prime}$ and $\beta$ in Table 1 are not optimal.

Remark 2 The improved Hardy-Rellich inequality (3.0) is crucial to prove that $u^{*}$ is singular in dimensions $n \geq 13$. Indeed by the classical Hardy-Rellich inequality and $u:=w_{2}$, Lemma 5.4 only implies that $u^{*}$ is singular $n$ dimensions $n \geq 32$.

Acknowledgements. The first author would like to thank his advisor Prof. Yi-Li for his constant support, and encouragement. This research is supported in part by National Natural Science Foundation of China (Grant No. 10971061).

\section{References}

[1] M. G. Crandall, P. H. Rabinawitz, Some continuation and variational methods for positive solutions of nonlinear elliptic eigenvalue problems, Arch. Ration. Mech. Anal., 58 (1975), 207-218.

[2] D.D. Joseph, T. S. Lundgren, Quasilinear Dirichlet problems driven by positive sources, Arch. Ration. Mech. Anal., 49, (1973), 241-268.

[3] P. L. Lions, On the existence of positive solutions of semilinear elliptic equations, SIAM Rev., 24, (1982), 441-467.

[4] Y. Martel, Uniqueness of weak extremal solutions for nonlinear elliptic problems, Houston J. Math., 23, 161-168 (1997).

[5] F. Mignot, J. P. Puel, Solution radiale singulière de $-\Delta u=\lambda e^{u}$, C. R. Acad. Sci. Paris Sér. I 307, (1988), 379-382.

[6] E. Berchio, Gazzola, F., Some remarks on biharmonic elliptic problems with positive, increasing and convex nonlinearities. Electronic J. Differ. Equ. 34 (2005), 1-20.

[7] A. Ferrero, H.-C. Grunau, The Dirichlet problem for supercritical biharmonic equations with powertype nonlinearity, J. Differ. Equ. 234 (2007), 582C606.

[8] A. Ferrero, H.-C. Grunau, P. Karageorgis, Supercritical biharmonic equations with powertype nonlinearity, Annali di Matematica 188, (2009), 171-185.

[9] J. Dàvila, I. Flores, I. Guerra, Multiplicity of solutions for a fourth order equation with power-type nonlinearity, Math. Ann. 348, (2009), 143-193.

[10] T. Boggio, Sulle funzioni di Freen dordine m. Rend. Circ. Mat. Palermo 20 (1905), 97-135.

[11] G. Arioli, F. Gazzola, H.-C. Grunau, E. Mitidieri, A semilinear fourth order elliptic problem with exponential nonlinearity. Siam J. Math. Anal. 36, (2005), 1226-1258.

[12] Brezis, H., Vazquez, J. L., Blow up solutions of some nonlinear elliptic problems. Rev. Mat. Univ. Complutense Madrid 10, (1997), 443-469.

[13] N. Ghoussoub, A. Moradifam, Bessel pairs and optimal Hardy and Hardy-Rellich inequalities, Submitted (2008).

[14] J. J. Moreau, Décomposition orthogonale d'un espace hilbertien selon deux cones mutuellement polaires, C. R. Acad. Sci. Paris 255 (1962), 238-240. 
[15] C. Cown, P. Esposito, N. Ghoussoub, and A. Moradifam, The critical dimension for a forth order elliptic problem with singular nonlineartiy, Arch. Ration. Mech. Anal., (2010, to appear).

[16] A. Moradifam, The singular extremal solutions of the bi-laplacian with exponential nonlinearity Proc. Amer. Math. Soc., 138 (2010) 1287-1293.

[17] J. Dàvila, L. Dupaigne, I. Guerra, and M. Montenegro, Stable Solutions for the Bilaplacian with Exponential nonlinearity, SlAM J. Math. Anal., 39 (2007), 565-592. 
Table 1

\begin{tabular}{|l|l|l|}
\hline$n$ & $\lambda^{\prime}$ & $\beta$ \\
\hline 31 & $3.06 K_{0}$ & $4.05 K_{0}$ \\
\hline $30-19$ & $4.6 K_{0}$ & $10 K_{0}$ \\
\hline 18 & $3.5 K_{0}$ & $3.78 K_{0}$ \\
\hline 17 & $3.26 K_{0}$ & $3.60 K_{0}$ \\
\hline 16 & $3.13 K_{0}$ & $3.78 K_{0}$ \\
\hline 15 & $2.76 K_{0}$ & $3.12 K_{0}$ \\
\hline 14 & $2.34 K_{0}$ & $2.96 K_{0}$ \\
\hline 13 & $2.03 K_{0}$ & $2.15 K_{0}$ \\
\hline
\end{tabular}

